\title{
How much can a small bursa stretch? A curious case of massive superficial infrapatellar bursitis in the emergency department
}

\author{
Ganesh Singh Dharmshaktu ${ }^{1^{*}(\mathbb{D}}$, Tanuja Pangtey $^{2}$ \\ 'Department of Orthopaedics, Government Medical College, Haldwani, Uttarakhand, India \\ 2Department of Pathology, Government Medical College, Haldwani, Uttarakhand, India
}

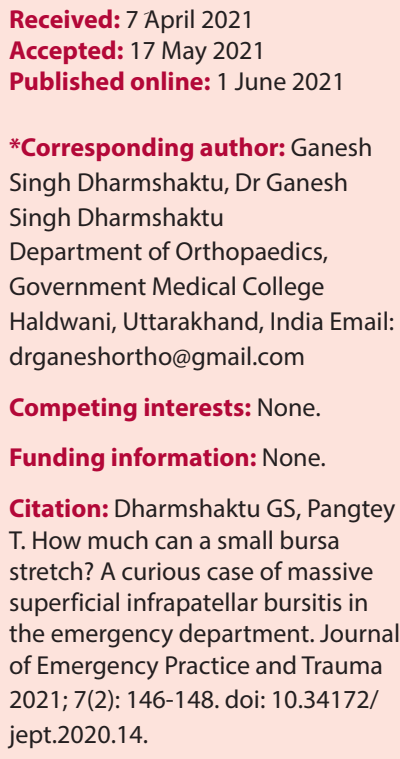

\begin{abstract}
Introduction: Inflammation of the infrapatellar bursa results from various causes like trauma, infection and overuse. Superficial skin or soft tissue infection can also lead to occasional septic bursitis. Infrapatellar bursa has two parts, superficial and deep bursae. Superficial bursa inflammation reactive to adjacent superficial skin infection may occasionally lead to secondary bursitis.

Case Presentation: We report a rare finding of acute right knee pain with extraordinary amount of fluid collection within superficial part of infrapatellar bursa in an elderly male patient. A secondary, septic and massive superficial infrapatellar bursitis was unusual in its appearance mimicking neoplastic lesion. Judicious use of clinical assessment and imaging helped to diagnose the condition.

Conclusion: Early diagnosis and treatment of bursitis can ensure optimal outcome and it can be used to check further complications. Atypical presentation of any bursitis should be acknowledged and the judicious use of clinical assessment and imaging is critical for prompt diagnosis and treatment.

Keywords: Bursa, Infrapatellar bursa, Clergyman's knee, Knee swelling
\end{abstract}

\section{Introduction}

Infra-patellar bursa, situated adjacent to distal patellar tendon insertion, is one of the many bursae around the knee (1). The 'clergymen's knee' is a colloquial term used to describe its inflammation as the condition is found commonly in priests following frequent kneeling postures. This bursa has superficial and deep parts and the former one lies over the patellar tendon and is commonly involved in bursitis. A well-defined palpable swelling is the common presentation, the size and extent of which can be confirmed on magnetic resonance imaging (MRI). MRI can not only distinguish it from subcutaneous edema but also differentiates superficial from deep bursitis. The bursitis commonly results from trauma, overuse, hemorrhage or infection with less common causes including vascular, inflammatory arthropathies or collagen disorders (2). Usually small swelling is noted in the infra-patellar region and a massive growth resembling a neoplasm is a rare presentation.

\section{Case Presentation}

A 60-year old male, farmer by occupation, with a huge swelling below his right knee for a duration of one week was referred to our emergency department. The swelling was atraumatic, mildly painful, not limiting the knee movement or activities of daily living, minimal to begin with but increasing in size over the week that made him consult a doctor (Figure $1 \mathrm{a}-\mathrm{b})$. The swelling was below the patella, fluctuant, and well defined with normal overlying temperature. However, there was superficial cellulitis over lateral leg, contra-lateral leg and adjacent to the swelling during the last eight days with no treatment taken (Figure 1c). The knee MRI revealed a large fluid collection in the superficial infrapatellar bursa without any bony afflictions around the knee (Figures 2 and 3). The aspiration resulted in $120 \mathrm{~mL}$ of non-purulent turbid fluid which was sent for serological tests along with relevant blood tests including hemoglobin, total and differential counts, C-reactive protein and erythrocyte sedimentation rate. The above blood investigations 


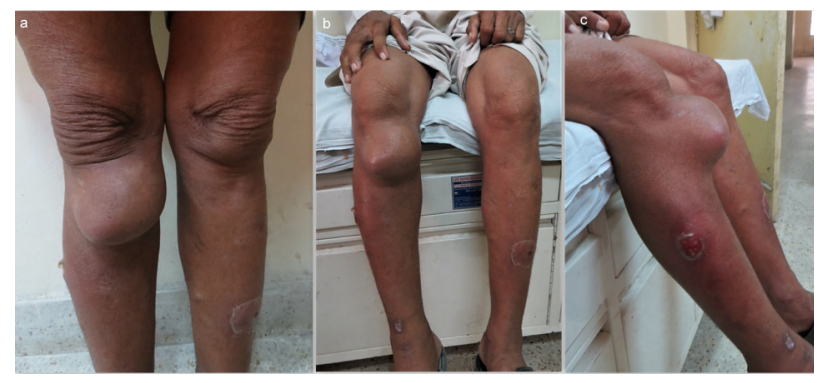

Figure 1. The clinical picture showing a huge swelling below right patella from front standing (a) and on sitting (b). The swelling is associated with adjacent cellulitis in lateral leg (c).

were unremarkable except raised total leukocyte count of $18000 / \mathrm{mm}$. The cell counts of the aspirate was found to be $53000 / \mathrm{mm}$ with polymorphonuclear leukocyte predominance. Culture of the aspirate for Gram, acid fast bacillus staining and polymerase chain reaction for tuberculosis were negative. On clinical basis along with increased cell counts, the diagnosis of septic bursitis was made secondary to overlying cellulitis. Cold fomentation, rest to the limb in the knee brace, pain medication as per the requirement and empirical antibiotic therapy with intravenous ceftriaxone 1 gram resulted in recovery. In addition, amikacin $500 \mathrm{mg}$ twice a day for a week followed by oral cefuroxime $500 \mathrm{mg}$ twice a day for two weeks led to complete recovery over a period of three weeks with no recurrence in the follow up of nine months.

\section{Discussion}

Infection directly or adjacent to any bursa can lead to septic bursitis and can present acutely or in chronic recurrent fashion. Overlying superficial infection is a described trigger for secondary infectious bursitis (3). The signs of infection may be subtle at times thus making biochemical tests of aspirate crucial to diagnosis. In our case, there was adjacent skin infection that might have resulted in septic bursitis but the signs of infection were not remarkable over the bursa. Early treatment results in uneventful recovery and checks its progression into septic arthritis (4). As only superficial part of infrapatellar bursa was involved in our case and timely diagnosis was made, the dreaded complication of septic knee arthritis was avoided. The most common pathogens identified with septic bursitis are Staphylococcus aureus and other gram positive organisms, but gram negative ones and fungi are also noted in sporadic case reports (5). The superficial bursa, however, is not visualized in every knee as found in a study done with ultrasound guided bursography in cadaveric knees. It may occasionally have a septum separating its compartments (6). The huge collection within an otherwise less spacious bursa reveals the bearing capacity of human tissue and the massive collection may mimic neoplastic lesion at initial glance. The massive size
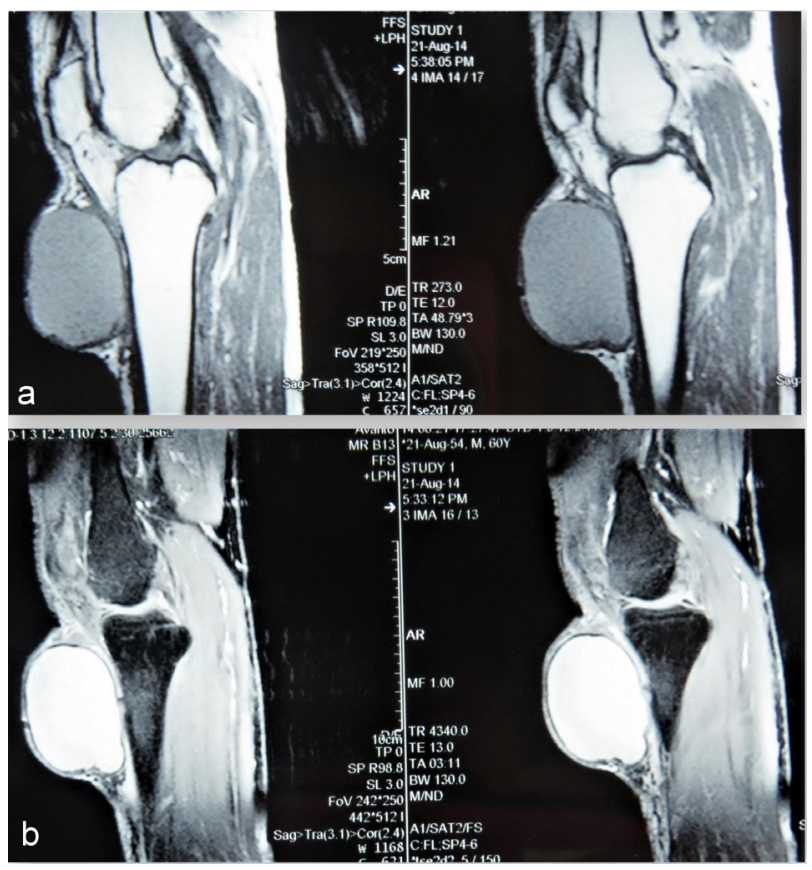

Figure 2. The MRI images showing the large bursa collection in saggital plane $(a, b)$ depicting the infrapatellar bursitis.

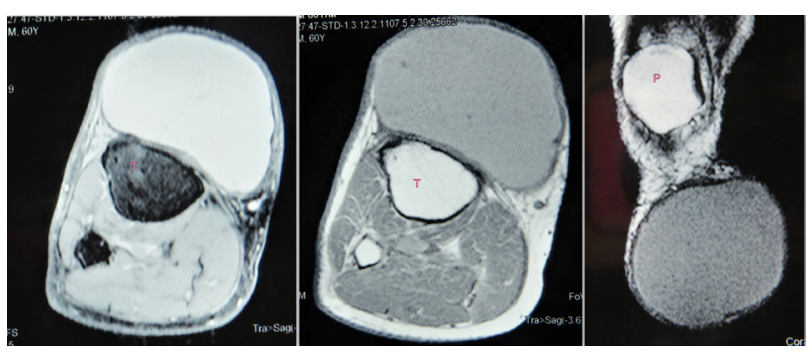

Figure 3. The MRI images of axial cuts inT1 and T2 weighted scans showing a large collection in front of tibia and the same lesion below the patella (Tibia denoted by $\mathrm{T}$ and patella by $\mathrm{P}$ ).

of bursitis at an uncommon location is unusual.

\section{Conclusion}

The striking clinical picture highlights the importance of knowledge of bursae around knee and judicious use of clinical, radiological and pathological methods to diagnose it for prompt management.

\section{Authors' contributions}

Each author contributed equally to study design, drafting the article, reading critically and accepting the final proof.

\section{Ethical issues}

Informed consent was obtained from the patient for publishing this report.

\section{References}

1. Chatra PS. Bursae around the knee joints. Indian J 
Radiol Imaging 2012; 22(1): 27-30. doi: 10.4103/09713026.95400.

2. McCarthy CL, McNally EG. The MRI appearance of cystic lesions around the knee. Skeletal Radiol 2004; 33(4): 187-209. doi: 10.1007/s00256-003-0741-y.

3. Truong J, Ashurst JV. Septic Bursitis. In: StatPearls. Treasure Island, FL: StatPearls Publishing; 2019.

4. Lieber SB, Fowler ML, Zhu C, Moore A, Shmerling $\mathrm{RH}, \mathrm{Paz}$ Z. Clinical characteristics and outcomes of septic bursitis. Infection 2017; 45(6): 781-6. doi: 10.1007/s15010-017-1030-3.

5. Zimmermann B 3rd, Mikolich DJ, Ho G Jr. Septic bursitis. Semin Arthritis Rheum 1995; 24(6): 391410. doi: 10.1016/s0049-0172(95)80008-5.

6. Viegas FC, Aguiar RO, Gasparetto E, Marchiori E, Trudell DJ, Haghighi P, et al. Deep and superficial infrapatellar bursae: cadaveric investigation of regional anatomy using magnetic resonance after ultrasound-guided bursography. Skeletal Radiol 2007; 36(1): 41-6. doi: 10.1007/s00256-006-0142-0. 